\title{
Evaluation of a weight management programme delivered onboard a warship
}

\author{
A.M. Shaw ${ }^{1,2}$, L. Morrow ${ }^{1}$, C. Abrams $^{1}$, S. Downie ${ }^{1}$, A.J. Allsopp ${ }^{1}$, E.L. Parsons ${ }^{2,3}$, \\ S.A. Wootton ${ }^{2,3}$ and J.L. Fallowfield ${ }^{1}$ \\ ${ }^{1}$ Institute of Naval Medicine, Alverstoke, Gosport, PO12 2DL, ${ }^{2}$ Human Development and Health Academic Unit, \\ Faculty of Medicine, University of Southampton, Southampton, SO17 1 BJ and ${ }^{3}$ NIHR Southampton \\ Biomedical Research Centre, Southampton Centre for Biomedical Research, Southampton General Hospital, \\ Southampton, SO16 $6 Y D$.
}

The United Kingdom Armed Forces are not exempt from the obesity epidemic ${ }^{(1)}$. This is of concern due to the health, occupational and economic risks that it poses. In response to this problem, a nine-month healthy lifestyle intervention was implemented aboard a deployed Royal Navy (RN) warship in 2015. One component of the intervention was the delivery of a weight management programme (WMP). The present study evaluated the effectiveness of this WMP in accordance with best practice guidelines for weight management services ${ }^{(2)}$.

The delivery and content of the WMP was based on the National Institute for Health and Care Excellence (NICE) guidance for the provision of weight management services ${ }^{(3)}$. The programme was available for all members of the ship's company $(n 242)$ who wanted to improve their health behaviours, rather than just those classified at risk of obesity related diseases according to the NICE guidelines ${ }^{(4)}$. Personnel with a body mass index of less than $18.5 \mathrm{~kg} \cdot \mathrm{m}^{-2}$ (i.e. underweight) were not allowed to participate in the programme.

Forty-nine RN and Royal Marines personnel (males $84 \%$, females $16 \%$ ), mean (SD) age 31 (7) years attended the WMP for the duration of the deployment. This equated to $20 \%$ of the ship's company. Participants' height, body weight and waist circumference were measured at three time points; pre-, mid- (4-5 months) and end- ( 9 months) of deployment.

The WMP adherence rate was $100 \%$ (i.e. no drop outs). At pre-deployment, $45 \%$ of participants were classified at any risk of obesity related diseases $(12 \%$ increased risk, $14 \%$ high risk, $18 \%$ very high risk). This reduced to $29 \%$ at end-deployment $(10 \%$ increased risk, $14 \%$ high risk, $4 \%$ very high risk). There was a significant decrease in (mean [SD]) weight (91.8 [16.6] vs. 86.6 [14.8] kg, P<0.001] and waist circumference $(93.4$ [12.8] vs. 85.7 [9.8] cm, $\mathrm{P}<0.001)$ between pre- and end-deployment. Weight loss over the deployment was $5.2[4.7] \%$ of initial weight $(\mathrm{P}<0.001)$. Weight and waist circumference reduction was greatest between pre- and mid-deployment, with smaller reductions between mid- and end-deployment. There was a medium negative correlation between relative weight loss and waist circumference reduction during the deployment and weight and waist circumference measured pre-deployment $(\mathrm{r}=0.51, \mathrm{P}<0.001 ; \mathrm{r}=0.52, \mathrm{P}<0001$, respectively). The WMP therefore appeared to be more successful for participants at higher risk at pre-deployment. At the end of deployment $65 \%$ and $61 \%$ of participants achieved a weight loss greater than $3 \%$ and $5 \%$ of their initial weight, respectively; this exceeded the Department of Health best practice guidance for Tier-2 services ${ }^{(2)}$.

The findings from the present study support that an effective WMP can be delivered in a deployed military environment. Support should be offered to all personnel as prevention, rather than just being available as treatment to those classified at risk of obesity related diseases.

C Crown Copyright (MOD) 2018: Published with the permission of the Controller of HMSO.

1. Wood P (2007) Dstl Report No.: TR27252. Dstl, Farnborough, UK.

2. Department of Health (2013) Developing a specification for lifestyle weight management services. Department of Health, London, UK.

3. NICE (2014). Managing overweight and obesity in adults - lifestyle weight management services PH53. NICE, London, UK.

4. NICE (2014) NICE guidelines CG189. NICE, London, UK. 